\title{
Modelos Matemáticos para Manejo Integrado da Grapholita molesta na Cultura do Pessegueiro
}

\section{Elisa Regina Cara ${ }^{1}$}

Programa de Pós-Graduação em Matemática Aplicada, UFRGS, Porto Alegre, RS,

\section{Maria Cristina Varriale ${ }^{2}$}

Departamento de Matemática Pura e Aplicada, UFRGS, Porto Alegre, RS

Resumo A Grapholita molesta (mariposa oriental), na cultura do pessegueiro na Região Sul do Brasil é uma praga que provoca perdas na produção da ordem de $3 \%$ a $5 \%$. Diante disso, desenvolvemos um modelo matemático para o seu controle, envolvendo três fases do ciclo de vida da mariposa e dois inimigos naturais (parasitoides). Determinamos os pontos de equilíbrio do modelo e suas respectivas estabilidades. Adotamos o Manejo Integrado de Pragas (MIP), incluindo controles biológico e químico, a fim de manter a praga abaixo do Limiar Econômico (LE). O controle biológico foi adotado através da liberação de parasitoides de ovos Trichogramma pretiosum e de parasitoides de lagartas Macrocentrus ancylivorus; o controle químico através da utilização de inseticidas seletivos. Trabalhamos inicialmente com o modelo sem estrutura espacial, para o qual adotamos na sequência uma abordagem de Redes de Mapas Acoplados, com dispersão por difusão e por taxia quase local. Resultados são visualizados através de gráficos das soluções numéricas.

Palavras-chave. mariposa oriental, Manejo Integrado de Pragas, Limiar Econômico, controle.

\section{Introdução}

A Grapholita molesta (Lepidoptera: Tortricidae) ou mariposa oriental, é uma das principais pragas associadas à cultura do pessegueiro na Região Sul do Brasil [4]. A ocorrência da mariposa oriental está relacionada ao cultivo de frutíferas da família Rosaceae como pessegueiros, ameixeiras, macieiras, dentre outras. Trata-se de um inseto com metamorfose completa, passando pelas fases de ovo, lagarta, pupa e adulta. As perdas na produção se dão na fase de lagarta, pois esta alimenta-se dos ponteiros e frutos, inviabilizando-os para o consumo [4].

\footnotetext{
${ }^{1}$ elisa.regina@ufrgs.br

${ }^{2}$ cristina.varriale@ufrgs.br
} 
O controle da mariposa oriental é feito, quase que totalmente, através da aplicação de inseticidas químicos de amplo espectro (fosfarados e peritroides), os quais são altamente tóxicos, tanto para a praga, quanto para o meio ambiente em geral [4]. Com o objetivo de reduzir a utilização de inseticidas químicos e adotar técnicas que sejam benéficas ao meio ambiente, saúde dos consumidores e ao mesmo tempo eficazes para o controle da praga, desenvolvemos um modelo matemático que descreve a interação entre três fases do ciclo de vida da mariposa (ovo, lagarta e adulto), o parasitoide de ovos Trichogramma pretiosum e o parasitoide de lagartas Macrocentrus ancylivorus. Incorporamos a esse modelo estratégias de controle biológico através da liberação de parasitoides; o controle químico, através da aplicação de inseticidas seletivos.

O Manejo Integrado de Pragas (MIP) surgiu para enfatizar a necessidade da incorporação de conceitos básicos de ecologia no desenvolvimento e implementação de sistemas de controle de pragas [5]. Ele consiste na utilização simultânea de diferentes técnicas de supressão populacional como estratégia de combate à praga, de modo a manter a população de insetos em uma condição de não praga (que não causam danos) de forma econômica e harmônica com o ambiente [5]. Desta forma, o que se deseja não é erradicar completamente a população de pragas, mas sim reduzí-la a níveis toleráveis, abaixo de um Limiar Econômico (LE) ou Nível de Controle (NC).

Muitos trabalhos têm sido desenvolvidos [3,6,7], explorando a aplicação de técnicas do MIP em modelos presa-predador. Adicionalmente, incorporamos uma estrutura espacial ao modelo matemático desenvolvido, através de uma Rede de Mapas Acoplados, na qual consideramos dispersão por difusão e por taxia quase local.

\section{Modelo Básico Adimensional}

O modelo básico para a dinâmica populacional da mariposa oriental e de seus principais inimigos naturais é composto pelas seguintes cinco EDO's de primeira ordem não lineares:

$$
\left\{\begin{array}{l}
\frac{d v}{d t}=\gamma n(1-n)-\left(\alpha_{1}+\mu_{1}\right) v-\frac{\beta_{1} v s}{1+v}, \\
\frac{d l}{d t}=\alpha_{2} v-\left(\alpha_{3}+\mu_{2}\right) l-\frac{\beta_{2} l p}{1+l}, \\
\frac{d n}{d t}=\alpha_{4} l-\mu_{3} n, \\
\frac{d s}{d t}=\frac{\beta_{3} v s}{1+v}-\mu_{4} s, \\
\frac{d p}{d t}=\frac{\beta_{4} l p}{1+l}-\mu_{5} p,
\end{array}\right.
$$

onde $v(t), l(t), n(t), s(t)$ e $p(t)$ representam as populações adimensionais (razão entre as populações originais e a capacidade suporte de cada uma) de ovos, lagartas, mariposas adultas, parasitoides de ovos e parasitoides de lagartas, respectivamente; quanto aos parâmetros adimensionais, $\gamma$ é a taxa de crescimento intrínseca da população $v(t)$; $\alpha_{i}, i=1, \ldots, 4$ são parâmetros de conversão entre as três fases da mariposa oriental; $\mu_{i}$, $i=1, \ldots, 5$ são taxas de morte natural das populações e $\beta_{i}, i=1, \ldots, 4$ são coeficientes de 
parasitismo entre parasitoides e seus respectivos hospedeiros. O parasitismo se dá através da Resposta Funcional de Holling Tipo II, que é a que melhor descreve as interações parasitoide-hospedeiro [6].

\subsection{Pontos de Equilíbrio e Viabilidade Biológica}

Obtivemos os seguintes cinco pontos de equilíbrio:

- Extinção de todas as populações: $E_{0}=(0 ; 0 ; 0 ; 0 ; 0)$;

- Extinção de ambos os predadores: $E_{1}=\left(v_{1} ; l_{1} ; n_{1} ; 0 ; 0\right)$;

- Extinção do predador de ovos: $E_{2}=\left(v_{2} ; l_{2} ; n_{2} ; 0 ; p_{2}\right)$;

- Extinção do predador de lagartas: $E_{3}=\left(v_{3} ; l_{3} ; n_{3} ; s_{3} ; 0\right)$;

- Coexistência de todas as espécies: $E_{4}=\left(v_{4} ; l_{4} ; n_{4} ; s_{4} ; p_{4}\right)$, onde:

$$
\begin{aligned}
& v_{4}=\frac{\mu_{4}}{\beta_{3}-\mu_{4}} ; \quad l_{4}=\frac{\mu_{5}}{\beta_{4}-\mu_{5}} ; \quad n_{4}=\frac{\alpha_{4} \mu_{5}}{\mu_{3}\left(\beta_{4}-\mu_{5}\right)} ; \\
& s_{4}=\frac{\gamma \beta_{3} \alpha_{4} \mu_{5}}{\beta_{1} \mu_{3} \mu_{4}\left(\beta_{4}-\mu_{5}\right)}\left[1-\frac{\alpha_{4} \mu_{5}}{\mu_{3}\left(\beta_{4}-\mu_{5}\right)}\right]-\frac{\left(\alpha_{1}+\mu_{1}\right) \beta_{3}}{\beta_{1}\left(\beta_{3}-\mu_{4}\right)} ; \\
& p_{4}=\frac{\beta_{4}}{\beta_{2}}\left[\frac{\alpha_{2} \mu_{5}}{\mu_{5}\left(\beta_{3}-\mu_{4}\right)}-\frac{\left(\alpha_{3}+\mu_{2}\right)}{\beta_{4}-\mu_{5}}\right] .
\end{aligned}
$$

Dentre os equilíbrios citados acima, nosso interesse reside no equilíbrio $E_{4}$, de coexistência das espécies. Este equilíbrio é biologicamente viável se as seguintes quatro condições estiverem satisfeitas:

$$
\begin{aligned}
& \mu_{4}<\beta_{3} ; \quad \mu_{5}<\beta_{4} ; \quad R_{1}=\frac{\left(\beta_{3}-\mu_{4}\right)\left(\alpha_{3}+\mu_{2}\right)}{\left(\beta_{4}-\mu_{5}\right) \alpha_{2}}<1 ; \\
& R_{2}=\frac{\left(\alpha_{1}+\mu_{1}\right)\left(\beta_{4}-\mu_{5}\right)^{2} \mu_{4} \mu_{3}^{2}}{\gamma \alpha_{4} \mu_{5}\left(\beta_{3}-\mu_{4}\right)\left(\mu_{3}\left(\beta_{4}-\mu_{5}\right)-\alpha_{4} \mu_{5}\right)}<1 .
\end{aligned}
$$

As condições de estabilidade local para os pontos de equilíbrio também foram obtidas, utilizando o Critério de Routh-Hurwitz [1].

\subsection{Escolha dos Parâmetros do Modelo e Simulações Numéricas Iniciais}

No que segue, adotamos para os parâmetros do modelo (1): $\gamma=2,5, \mu_{1}=0,205$, $\mu_{2}=0,051, \mu_{3}=0,042, \mu_{4}=0,066, \mu_{5}=0,039, \alpha_{1}=0,656, \alpha_{2}=0,492, \alpha_{3}=0,713$, $\alpha_{4}=0,205, \beta_{1}=0,33, \beta_{2}=0,574, \beta_{3}=0,312$ e $\beta_{4}=0,459$, valores calculados a partir de dados experimentais obtidos por $[2,4]$. Os valores atribuídos aos parâmetros satisfazem as condições de viabilidade biológica (2) e estabilidade local para a coexistência das espécies.

Através das simulações numéricas verificamos o comportamento da mariposa oriental e dos parasitoides sem a aplicação de nenhum tipo de controle. Na Figura 1 apresentamos os comportamentos das cinco populações, obtidas por métodos numéricos (Runge-Kutta de $4^{\text {a }}$ Ordem) ainda sem aplicação de nenhum controle. Observamos que todas as populações tendem ao equilíbrio, cujos valores verificam as expressões obtidas na seção $2.1: E_{4}=$ $(0,2683 ; 0,0929 ; 0,4532 ; 5,5657 ; 1,2520)$. 


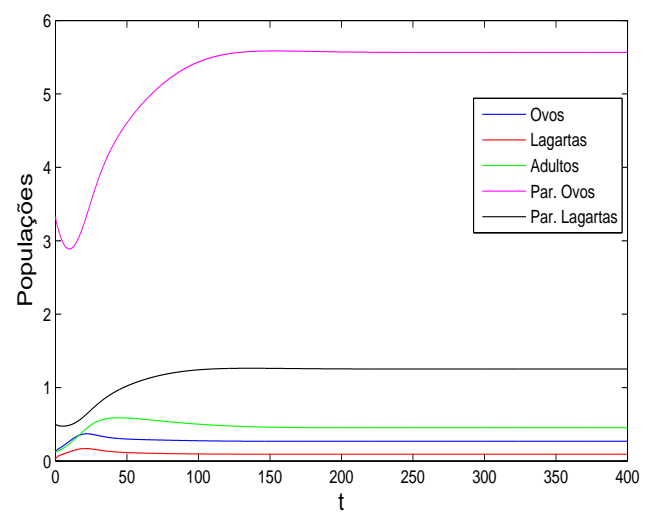

Figura 1: Populações tendendo ao ponto de equilíbrio de coexistência $E_{4}$.

\section{Modelo para o Controle da G. molesta Através do MIP}

O modelo matemático para o manejo integrado da $G$. molesta, considerando a liberação artificial de parasitoides e aplicação de inseticidas seletivos, toda vez que a população de mariposas adultas atinge ou ultrapassa o Limiar Econômico (LE) é dado por:

$$
\left\{\begin{array}{l}
v\left(t^{+}\right)=\left(1-m_{1}\right) v(t), \\
l\left(t^{+}\right)=\left(1-m_{2}\right) l(t), \\
n\left(t^{+}\right)=\left(1-m_{3}\right) n(t), \\
s\left(t^{+}\right)=s(t)+\eta, \\
p\left(t^{+}\right)=p(t)+\tau,
\end{array}\right.
$$

onde $v\left(t^{+}\right), l\left(t^{+}\right), n\left(t^{+}\right), s\left(t^{+}\right)$e $p\left(t^{+}\right)$denotam o número de ovos, lagartas, fêmeas adultas, parasitoides de ovos e parasitoides de lagartas, respectivamente, após cada aplicação do controle integrado, no tempo $t$. No modelo (3), as mortalidades $0 \leq m_{i}<1, i=1, \ldots, 3$, são a proporção pela qual a densidade de ovos, lagartas e adultos, respectivamente, é reduzida por morte devido à aplicação de inseticidas seletivos uma vez que a população de adultos atinge o LE. Além disso, $\eta \geq 0$ é o número constante de parasitoides de ovos e $\tau \geq 0$ é o número constante de parasitoides de lagartas liberados no tempo $t$.

$\mathrm{Na}$ Figura 2, supomos que o inseticida fosse um lagarticida $\left(m_{2}=0,85\right)$, por isso não mata os ovos $\left(m_{1}=0\right)$, e é pouco prejudicial aos adultos $\left(m_{3}=0,5\right)$. Fixamos a liberação de parasitoides de ovos em $\eta=1$ e a liberação de parasitoides de lagartas em (a) $\tau=1$ e (b) $\tau=2$. Das diversas atribuições aos parâmetros envolvidos no sistema (3), os que correspondem a Figura 2, evidenciam os seguintes fatos: conforme a quantidade de parasitoides de lagartas liberados $\tau$ aumenta, menos aplicações do MIP são necessárias; a população de lagartas é reduzida sempre a mesma quantidade após cada aplicação do MIP. Além disso, ao recuperar-se, atinge proporções menores conforme a quantidade de parasitoides de lagartas aumenta. Desta forma, concluímos que o MIP é eficiente no controle das lagartas e que após os transientes iniciais, as aplicações do MIP são aparentemente periódicas, fato este que também foi comprovado por [6]. 


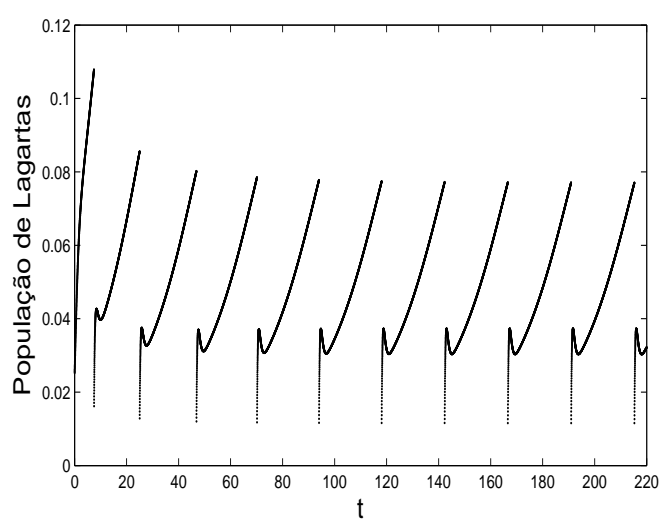

(a)

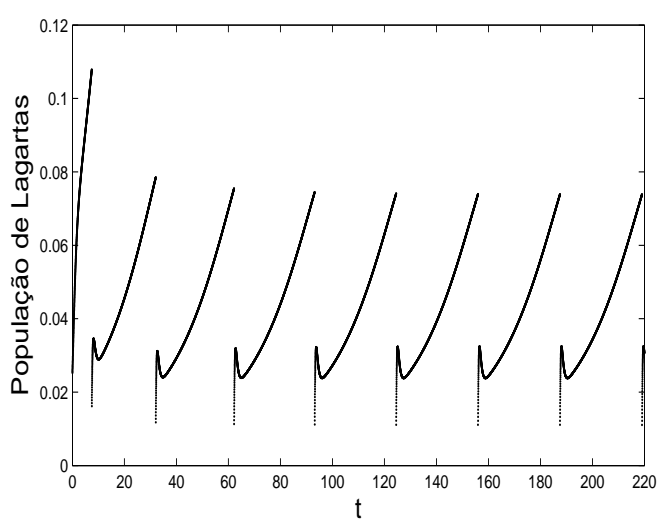

(b)

Figura 2: Variação na população de lagartas $l(t)$ com a aplicação do MIP com $m_{1}=0, m_{2}=0,85$, $m_{3}=0,5, L E=0,2$ para: (a) $\tau=1$ e (b) $\tau=2$.

\section{Modelo Espacialmente Estruturado para o Controle da G. molesta}

Incorporamos ao modelo adimensional (1), através de Redes de Mapas Acoplados, a movimentação das populações entre os sítios de uma vizinhança. O habitat é representado por uma malha (matriz) bidimensional de tamanho $55 \times 55$ sítios. Apenas os ovos da mariposa não se movimentam e a movimentação das lagartas é mais limitada, movimentando-se até os quatro vizinhos mais próximos. As mariposas adultas e os parasitoides podem se dispersar até os oito vizinhos mais próximos. A dinâmica do modelo com dispersão ocorre em duas etapas distintas: uma fase de movimentação, em que ocorre a dispersão das lagartas, fêmeas adultas, parasitoides de ovos e parasitoides de lagartas e, uma fase de reação, em que ocorre a dinâmica vital (reprodução, mortes e predação), descrita pelo modelo (1).

Considerando $v_{x}(t)$ e $c_{x}(t)$, para $c=l, n, s, p$, respectivamente, as densidades de ovos, lagartas, fêmeas adultas, parasitoides de ovos e parasitoides de lagartas no sítio $x$, no instante $t$, antes da dispersão; $v_{x}^{\prime}(t)$ e $c_{x}^{\prime}(t)$, para $c^{\prime}=l^{\prime}, n^{\prime}, s^{\prime}, p^{\prime}$, as densidades populacionais no sítio $x$, no instante $t$, após a dispersão, escrevemos as equações para a etapa de dispersão, como segue:

$$
\left\{\begin{array}{l}
v_{x}^{\prime}(t)=v_{x}(t), \\
c_{x}^{\prime}(t)=c_{x}(t)-\sum_{y \in V_{x}} S_{y}^{c}(t)+\sum_{y \in V_{x}} E_{y}^{c}(t),
\end{array}\right.
$$

onde $S_{y}^{c}(t)$ representam as populações que saem do sítio $x$ e vão para cada um dos sítios $y \in V_{x}$, a cada etapa de tempo $t ; E_{y}^{c}(t)$ representam as populações que entram no sítio $x$, oriundas de cada um dos sítios $y \in V_{x}$, a cada etapa de tempo $t$. Após a etapa de movimentação, ocorre a dinâmica vital dentro de cada sítio da malha, descrita pelo sistema: 


$$
\left\{\begin{array}{l}
\frac{d v}{d t}=\gamma n^{\prime}\left(1-n^{\prime}\right)-\left(\alpha_{1}+\mu_{1}\right) v^{\prime}-\frac{\beta_{1} v^{\prime} s^{\prime}}{1+v^{\prime}} \\
\frac{d l}{d t}=\alpha_{2} v^{\prime}-\left(\alpha_{3}+\mu_{2}\right) l^{\prime}-\frac{\beta_{2} l^{\prime} p^{\prime}}{1+l^{\prime}} \\
\frac{d n}{d t}=\alpha_{4} l^{\prime}-\mu_{3} n^{\prime} \\
\frac{d s}{d t}=\frac{\beta_{3} v^{\prime} s^{\prime}}{1+v^{\prime}}-\mu_{4} s^{\prime} \\
\frac{d p}{d t}=\frac{\beta_{4} l^{\prime} p^{\prime}}{1+l^{\prime}}-\mu_{5} p^{\prime}
\end{array}\right.
$$

\subsection{Dispersão por Difusão e MIP}

Para a dispersão por difusão, onde $D_{i}, i=l, n, s, p$ são os coeficientes de difusão de cada população, usaremos em (4), as seguintes expressões:

$$
\begin{aligned}
& S_{y}^{l}(t)=D_{l} \frac{l_{x}(t)}{4}, S_{y}^{n}(t)=D_{n} \frac{n_{x}(t)}{8}, S_{y}^{s}(t)=D_{s} \frac{s_{x}(t)}{8}, S_{x}^{p}(t)=D_{p} \frac{p_{x}(t)}{8} \\
& E_{y}^{l}(t)=D_{l} \frac{l_{y}(t)}{4}, E_{y}^{n}(t)=D_{n} \frac{n_{y}(t)}{8}, E_{y}^{s}(t)=D_{s} \frac{s_{y}(t)}{8}, E_{y}^{p}(t)=D_{p} \frac{p_{y}(t)}{8} .
\end{aligned}
$$

Para a aplicação do MIP como descrito em (3), dividimos a malha de tamanho $55 \times 55$ sítios em 25 blocos de tamanho $11 \times 11$ sítios. Em cada passo de tempo, em todos os sítios de cada bloco, medimos a densidade de fêmeas adultas $n$. Se a densidade $n$ atingir o LE em $10 \%$ dos sítios, aplica-se o MIP em todos os sítios do bloco. Esse processo é feito em cada iteração temporal. Consideramos que inicialmente apenas o sítio central $x=(28,28)$ esteja ocupado, com densidades iniciais $v_{x}(0)=0,1, l_{x}(0)=0,025, n_{x}(0)=0,13, s_{x}(0)=3,3 \mathrm{e}$ $p_{x}(0)=0,5$, e o restante do reticulado esteja vazio.
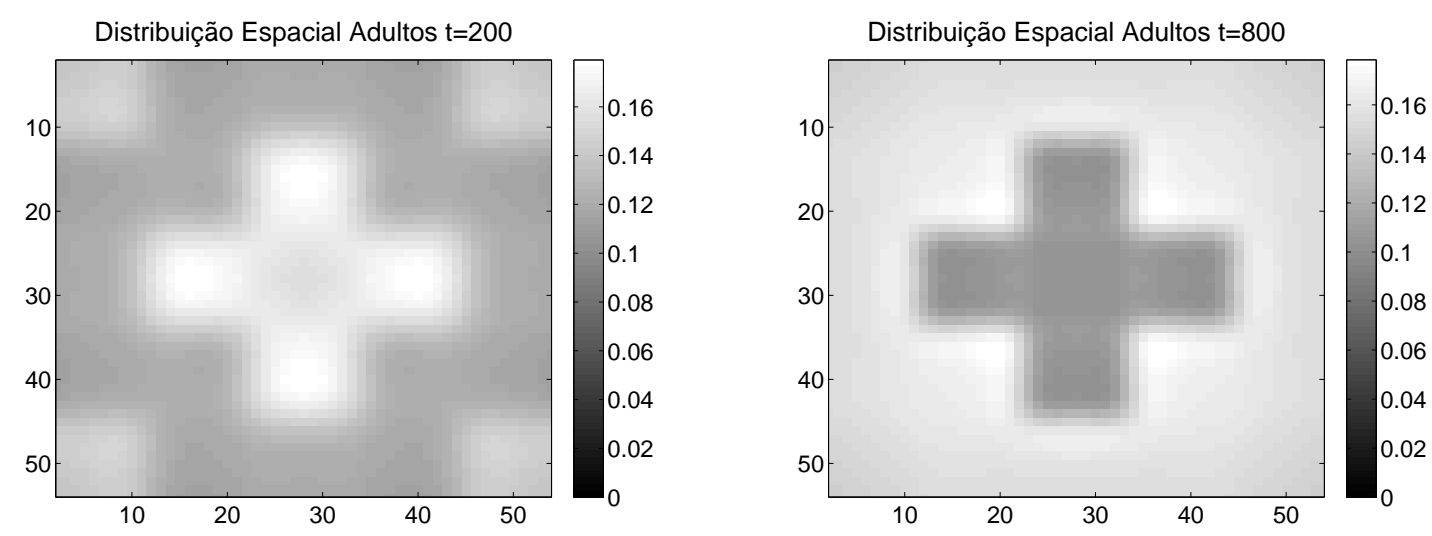

Figura 3: Distribuição espacial da população de fêmeas adultas $n$ com coeficiente de difusão $D_{n}=0,5$ e $L E=0,2$, nos instantes de tempo $t=200$ e $t=800$.

Na Figura 3, temos a distribuição espacial das fêmeas adultas apresentando padrões espaço-temporais heterogêneos. O MIP mostra-se eficiente para o controle da mariposa 
oriental no caso das populações espacialmente distribuídas, pois $n(t)$ é mantida abaixo do $L E=0,2$, em todos os sítios da malha (ver Figura 3). Resultados positivos também foram obtidos supondo que ambos os parasitoides se movimentem segundo um processo de taxia quase local e que as lagartas e fêmeas adultas se movimentem por difusão.

\section{Conclusões}

Os modelos apresentados para o controle da mariposa oriental utilizando o MIP mostraram-se eficientes, mantendo a densidade da praga em níveis toleráveis abaixo do LE, em todos os casos considerados. Além disso, as aplicações do MIP tornaram-se periódicas (Figura 2), o que é importante do ponto de vista da aplicação do controle, visto que o produtor não vai precisar medir a população da praga antes de cada aplicação do controle, passando a simplesmente aplicá-lo periodicamente.

No caso do modelo com distribuição espacial, o MIP provoca alterações no comportamento espaço-temporal das populações. Para a dispersão por difusão sem o MIP, a distribuição espacial das populações torna-se homogênea com o passar do tempo, mas ao aplicarmos o MIP (Figura 3), a distribuição espacial apresenta padrões heterogêneos instáveis. De forma geral, o manejo integrado da Grapholita molesta proposto neste trabalho mostrou-se uma boa alternativa na substituição das medidas de controle usualmente adotadas para a praga.

\section{Referências}

[1] L. J. S. Allen, An Introduction to Mathematical Biology, Pearson Prentice Hall, vol. $1,(2007)$.

[2] C. J. Arioli, M. S. Garcia, M. Zarte e M. Botton, Biologia da mariposa-oriental em dieta artificial à base de milho, Scientia Agraria, vol. 11, 481-486, (2010).

[3] S. Bhattacharyya and D. K. Bhattacharya, An improved integrated pest management model under 2-control parameters (sterile male and pesticide), Mathematical Biosciences, vol. 209, 256-281, (2007).

[4] M. Botton, D. E. Nava, C. J. Arioli, A. D. Grutzmacher e M. S. Garcia, Bioecologia, monitoramento e controle da mariposa-oriental na cultura do pessegueiro no Rio Grande do Sul, Circular Técnica Embrapa, vol. 86, Bento Gonçalvez, RS, (2011).

[5] R. F. Norris, E. P. Caswell-Chen and M. Kogan, Concepts in Integrated Pest Management, Prentice Hall, vol. 1, (2003).

[6] S. Tang and R. A. Cheke, Models of integrated pest control and their biological implications, Mathematical Biosciences, vol. 215, 115-125, (2008).

[7] S. Tang, Y. Xiao and R. A. Cheke, Multiple attractors of host-parasitoid models with integrated pest management strategies: Eradication, persistense and outbreak, Theoretical Population Biology, vol. 73, 181-197, (2008). 